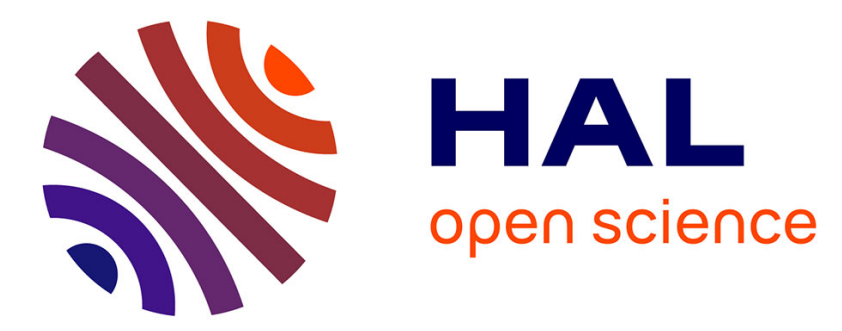

\title{
Une économie de la captation. Les dynamiques concurrentielles au sein du secteur funéraire
}

\author{
Pascale Trompette
}

\section{To cite this version:}

Pascale Trompette. Une économie de la captation. Les dynamiques concurrentielles au sein du secteur funéraire. Revue française de sociologie, 2005, 46 (2), pp.233-264. halshs-00311544

\section{HAL Id: halshs-00311544 https://shs.hal.science/halshs-00311544}

Submitted on 19 Aug 2008

HAL is a multi-disciplinary open access archive for the deposit and dissemination of scientific research documents, whether they are published or not. The documents may come from teaching and research institutions in France or abroad, or from public or private research centers.
L'archive ouverte pluridisciplinaire $\mathbf{H A L}$, est destinée au dépôt et à la diffusion de documents scientifiques de niveau recherche, publiés ou non, émanant des établissements d'enseignement et de recherche français ou étrangers, des laboratoires publics ou privés. 


\title{
Une économie de la captation
}

\section{Les dynamiques concurrentielles au sein du secteur funéraire}

\author{
Pascale Trompette*
}

\begin{abstract}
Résumé
La problématique de la rencontre entre offre et demande sur un marché est au cour du projet de la sociologie économique. Depuis plusieurs années, elle a donné lieu à l'analyse approfondie des modalités de construction du jugement sur les qualités du produit ou des personnes en amont de la relation d'échange. A partir de l'observation de la situation d'achat de services funéraires, conjuguée à l'analyse des dynamiques concurrentielles sur les marchés locaux, cet article met en évidence certaines modalités alternatives d'organisation de la rencontre marchande. Il décrit les dispositifs de captation comme la mise en æuvre d'agencements sociotechniques organisant la canalisation des clients vers un prestataire unique. Il montre à quel point la production de cette économie de la captation structure l'activité des opérateurs, les relations entre concurrents et le jeu de la régulation du marché.
\end{abstract}

\begin{abstract}
The problematic of how supply and demand meet on a particular market is at the core of the economic sociology project. For several years this problematic has generated detailed analyses of the ways in which judgments of product or service-provider quality are constructed before the exchange relation occurs. Through observation of how funeral services are purchased and analysis of competition dynamics on local funeral service markets, this article brings to light certain alternative ways of organizing the marketplace encounter in this business sector. It describes arrangements used to capture the market, such as socio-technical operations for channeling customers toward a single service provider. It shows the degree to which service-provider activity, relations among competitors, and the market regulation game in this sector are structured by moves to produce an economy based on client channelling.
\end{abstract}

\footnotetext{
* Je tiens à remercier Thomas Reverdy, Dominique Vinck, et tout particulièrement Pierre François ainsi que les membres du comité de rédaction de la revue, pour leurs précieuses remarques et suggestions sur les versions antérieures de ce texte.

Cet article est notamment tiré d'une étude réalisée dans le cadre du programme de recherche DARESPUCA « Services, Emploi, Territoires » (2000-2002). Olivier Boissin, économiste (LEPII - UPMF Grenoble II) a étroitement collaboré au travail d'enquête et de recherche qui portait plus largement sur la recomposition du marché dans le contexte de la suppression du monopole communal. La méthodologie de recherche a combiné des entretiens avec les directions d'entreprise et les représentants institutionnels du secteur (ministères, fédérations, consommateurs), l'observation directe de l'activité au sein des entreprises de pompes funèbres et en milieu hospitalier, l'étude de la réglementation et de la documentation juridique, ainsi que la participation à diverses instances de régulation du secteur (Conseil National des Opérations Funéraires, salons professionnels, activités syndicales, presse spécialisée). Pour des développements complémentaires sur cette recherche, cf. (Boissin, Trompette, 2004 ; Trompette, Boissin, 2000).
} 
Les développements récents de la sociologie économique ont produit une réflexion intense sur l'interface entre le client et le marché. En prenant appui sur l'économie des conventions (Boltanski, Thévenot, 1987), les recherches sociologiques ont parcouru une grande diversité de marchés pour identifier la variété des registres d'évaluation des biens (Favereau, 1989) ou des personnes (Eymard-Duvernay, Marchal, 1997) sur lesquels s'étaye la grammaire de la coordination. A partir de la généralisation du modèle dit «d'économie de la qualité » (Karpik, 1989), elles ont exploré de part en part l'activité de jugement et la pluralité des dispositifs permettant de surmonter l'opacité du marché ${ }^{1}$. De l'incertitude du marché à la complexité du calcul dans un espace de choix proliférant, la nouvelle sociologie du marché décrit finement les modalités d'encastrement sociocognitif du calcul comme activité distribuée (Callon, 1998 ; Callon, Muniesa, 2003 ; Cochoy, 2002). D’une approche à l'autre, le concept de qualification comme processus de définition des qualités du bien ou des personnes s'est progressivement imposé comme cet espace théorique fédérateur ${ }^{2}$ où se côtoient, se complètent et se concurrencent parfois ces nombreuses approches de l'intermédiation marchande.

Cette rapide revue des approches sociologiques du marché est loin d'être exhaustive, mais on peut néanmoins considérer que l'on évolue dans une représentation dominante de la rencontre marchande comme relevant d'un processus de jugement en amont de l'engagement marchand ou contractuel. Le processus de qualification peut certes être précédé par des apprentissages réciproques, coproduit au fil des échanges entre le client et le prestataire et cadré par des dispositifs sociotechniques (Dubuisson-Quellier, 1999) ${ }^{3}$, il n'en apparaît pas moins comme un moment liminaire

\footnotetext{
${ }^{1}$ Ces dispositifs sociotechniques se rapportent à un ensemble de formes personnelles (réseau social, qualité des personnes) et impersonnelles (agencements symbolico-matériels) participant à une mise en ordre de l'univers dans lequel se réalise le choix du consommateur ou à une orientation des principes d'action des cocontractants.

${ }^{2}$ Cf. Sociologie du travail 44(2002)-255-287. Dossier-débat : La qualité.

${ }^{3}$ Dans cette acception, l'offre et la demande ne préexistent pas à leur rencontre mais procèdent d'une co-construction par les partenaires, à travers des jeux d'anticipations, des processus d'échange et de négociations, des interactions et des apprentissages de la part des partenaires.
} 
de choix à partir duquel va s'accomplir (ou échouer) la rencontre des échangistes (De Bandt, Gadrey, 1994 ; Licoppe, et al., 2002) ${ }^{4}$.

L'observation de la situation d'achat de services funéraires, conjuguée à l'analyse des dynamiques concurrentielles sur les marchés locaux ${ }^{5}$, nous met en prise avec d'autres modalités d'organisation de la rencontre marchande, qui apparaissent pour le moins alternatives voire exclusives d'une quelconque forme de mise en scène du marché. En lieu et place de la prolifération des dispositifs propres à soutenir le travail de jugement, se déploient ici des agencements symbolico-matériels qui structurent la circulation du client et formatent rigoureusement la mise en relation avec un prestataire donné parmi une offre concurrentielle. En suivant de près l'itinéraire des clients sur le marché funéraire, nous verrons se dissoudre la frontière entre l'amont et l'aval de la relation d'échange, soit le temps de l'engagement contractuel et celui de la mise à disposition des biens et services. Cette situation invite à davantage prendre en considération le process dans la dynamique d'organisation du marché, comme dispositif central d'engagement et de captation du client.

L'article propose un développement en trois temps. Tout d'abord, nous nous intéresserons à l'acheteur de prestations funéraires («la famille», telle que la désignent communément les pompes funèbres), à ce moment essentiel où, immédiatement informé du décès d'un parent ou d'un proche, il se trouve au seuil du marché funéraire. Nous rencontrerons ici une figure sociale du client ${ }^{6}$ qui interpelle

\footnotetext{
${ }^{4}$ Cette lecture est particulièrement bien illustrée par les auteurs qui énoncent : «La consommation ordinaire est un processus. Celui-ci se déploie selon des séquences parmi lesquelles on peut distinguer trois moments génériques, celui de la préparation, celui de la transaction, et celui de l'usage des biens et des services. Ces trois étapes qui peuvent à l'occasion se chevaucher rejoignent en gros le découpage fonctionnel des économistes entre fonctions ex ante, transaction et fonction ex post » (Licoppe, et al., 2002, p. 101). On rencontre un raisonnement similaire, cette fois appliqué à l'économie des services, dans la contribution de A. Barcet et J. Bonamy à l'étude des relations et marchés de service (De Bandt, Gadrey, 1994, pp 153-174).

5 Même si la mobilité géographique et les bouleversements de la structure des familles ont sensiblement accru l'extension spatiale du parcours funéraire, les services funéraires demeurent des services de proximité. La structure du marché est basée sur des marchés locaux avec une forte concentration urbaine du fait de la distribution de la mortalité à partir des centres hospitaliers.

${ }^{6}$ Cf. le numéro spécial de la revue Sciences de la Société, coordonné par F. Cochoy, «Les figures sociales du client », $\mathrm{n}^{\circ} 56,2002$.
} 
particulièrement l'analyse sociologique (Cochoy, 2004 ; Karpik, 1989), à savoir un agent simultanément engagé dans un «achat impliquant » (prix et qualité de service) et en proie à l'égarement (opacité du marché, incertitude sur la qualité). De tels sujets marchands apparaîtront ici constitutifs d'une économie de la captation ${ }^{7}$ organisée par les opérateurs funéraires, dans la mesure où ils donnent prise aux dispositifs qui vont cadrer leur parcours sur le marché funéraire $(§ 1)$. L'étude du process de service comme lieu où s'organise la circulation du client occupera l'essentiel de notre second temps d'analyse. Nous envisagerons la façon dont les réseaux sociotechniques qui y sont mobilisés règlent étroitement les mouvements du défunt et de ses proches et canalisent la rencontre avec un opérateur privilégié. Ce constat nous amènera a identifier le process de service comme objet privilégié des stratégies et luttes concurrentielles dans le jeu de la régulation du marché (§2). Dans un troisième temps, nous examinerons de plus près les mécanismes d'enchaînement et d'engrenage qui jalonnent le parcours du client. Nous nous emploierons à appréhender plus finement les réseaux sociaux et les agencements symbolicomatériels qui supportent cette économie de la circulation sur le marché ainsi qu'à qualifier les dispositifs qui fabriquent l'engagement du client dans la relation d'échange (\$3).

\section{Un marché peuplé de « clients égarés »...}

Nous proposons d'entrer dans cette problématique de la rencontre entre une famille et un opérateur de pompes funèbres à partir de l'analyse des «dispositions » du client, comme préambule à celle des «dispositifs » (Cochoy, 2004, p.20-21) qui vont le conduire, le guider, le canaliser, tout au long du parcours qui le mène à une boutique de pompes funèbres. La sociologie du client sur le marché funéraire nous

\footnotetext{
${ }^{7}$ La notion de « captation » a récemment acquis une portée conceptuelle à part entière en sociologie du marché, notamment dans le cadre de la réflexion proposée et animée par F. Cochoy (Cochoy, 2004). Le projet des auteurs est sensiblement différent de celui adopté dans cet article. La catégorie de «captation » est mobilisée dans une acception large permettant d'englober et simultanément de visiter une multiplicité de modalités (attraction, séduction, fidélisation, sélection) et de dispositifs (emballage, commerce électroniques, scoring, etc.) organisateurs de la rencontre marchande. Nous préférons au contraire restreindre la notion de captation à l'idée de «canalisation contrainte », exclusive du jeu du marché. Cette distinction nous semble plus apte à différencier analytiquement les différents ressorts de la mise en relation des protagonistes de l'échange.
} 
amène à identifier une situation singulière : celle d'un client engagé dans un «achat impliquant », mais simultanément faible en habiletés de calcul et de jugement et confronté à un espace d'offres le plus souvent inconnu.

\subsection{Un achat impliquant}

Avant de partir directement à la rencontre de cet être quelque peu éprouvé que constitue le client des enseignes funéraires, il nous semble important de rappeler quelques-unes des propriétés qui caractérisent l'objet de la relation marchande, ne serait-ce que pour donner toute sa mesure au contraste entre le niveau d'engagement requis et les obstacles à la rationalisation du choix d'un prestataire.

La prestation funéraire se décrit comme une gamme de services engagés dans la transformation d'état du cadavre, à la fois dans l'ordre physiologique et symbolique. Autour du défunt, l'activité des pompes funèbres engage indissociablement des opérations techniques situées du côté du traitement du corps, des soins, du transport, $\mathrm{du}$ «conditionnement», et des opérations rituelles situées du côté du passage, de l'hommage et de la reconstruction familiale. L'achat de prestations funéraires peut être considéré comme un «achat impliquant ${ }^{8}$, au sens où il expose à des enjeux lourds, tant sur le plan financier que sur celui de la qualité de service. Si l'on s'attache en premier lieu à la composante économique, on peut estimer que les obsèques constituent une dépense conséquente, sinon très conséquente selon les contraintes budgétaires, avec un prix moyen établi aux alentours de 2500 à 2900 euros (hors concession et marbrerie) ${ }^{9}$. Si l'on se situe maintenant du côté des déterminants qualitatifs, on peut considérer que l'opérateur funéraire est un acteur essentiel des élaborations symboliques autour du défunt ${ }^{10}$. Figurants, accessoiristes et aujourd'hui metteurs en scène : les pompes funèbres sont les principaux interprètes

\footnotetext{
8 Nous faisons ici un usage à peine détourné de la notion «d'implication du consommateur » (involvment) dans l'achat de certaines catégories de produit, sur laquelle les sciences du marketing ont produit une abondante littérature (Zaichkowsky, 1985).

${ }^{9}$ Source : Etude de marché Xerfi. Services funéraires Secteurs 700, 2000.

${ }^{10}$ On se concentre ici sur les dimensions visibles de la prestation, en laissant de côté le problème du traitement et de la manipulation du cadavre. Cet enjeu est rarement évoqué par les familles, par l'effet conjugué du tabou social et de la méconnaissance des opérations se déroulant en back office.
} 
des rites d'hommage et de la mise en forme sacralisée et événementielle des funérailles, de l'un à l'autre des trois principaux temps de recueillement que sont la veillée, la procession et la cérémonie. Ils orchestrent la manifestation symbolique de la force du lien personnel, que ce soit sur le mode de l'expression publique et du faste ou, comme cela tendrait à apparaître aujourd'hui, sur celui de l'authenticité du lien au disparu et de l'émotion. En d'autres termes, on repère ici toutes les propriétés d'une «économie de la qualité » dans laquelle l'achat des biens et des services se raisonne dans l'ordre de l'incommensurable : manifester la profondeur de la perte et du deuil de «l'être cher» dans une relation à la fois intime (honorer sa dette) et publique (pratiquer un don noble propre à satisfaire au jugement social).

En conjuguant cet ensemble de caractéristiques, nous nous trouvons donc sur la voie d'une rencontre qui devrait s'organiser sous les «bons auspices » de la définition des qualités professionnelles des prestataires concurrents et de leur classement (Callon, Muniesa, 2003), en composant sur les variables prix et qualité selon la prégnance de la contrainte d'hommage qui anime le client. En d'autres termes, le commanditaire devrait, d'une façon ou d'une autre, travailler à se situer au mieux dans un espace d'offres différenciées, en prenant appui sur l'un ou l'autre des dispositifs propres à supporter le travail de jugement.

\subsection{Un marché illégitime}

Après avoir pris la mesure des propriétés susceptibles d'engager le client dans la quête du «bon croque-mort », nous prendrons celle des obstacles à la rationalisation de son choix. Cette problématique courante de la sociologie du marché nous convie ici à une situation relativement originale, cependant moins dans ses effets que dans ses fondements : celle d'un acteur rétif à la manifestation d'un marché jugé illégitime. En d'autres termes, les limites de ce marché concurrentiel tiennent moins aux conditions objectives d'organisation du marché (Garcia, 1986) qu'au tabou social qui pèse sur sa mise en scène comme marché. Ceci a au moins une conséquence majeure sur le problème de la rencontre entre clients et prestataires : 
celle de l'opacité de l'offre, qui résulte principalement du déficit de représentation publique des professionnels.

Le marché funéraire est un marché socialement illégitime (Thompson, 1991). Tout d'abord, parce que le «croque-mort » est une figure professionnelle marginalisée. Même en passant du statut de marchand à celui de professionnel des services funéraires, les métiers du funéraire ont conservé un arrière-goût de déclassement social. Au compagnonnage des cadavres, les croque-morts ajoutent la sanction morale d'oser tirer quelque bénéfice économique du commerce des biens symboliques (Henaff, 2002). Ce tabou contrarie l'entrepreneur funéraire en situation de se mettre en scène pour devenir l'heureux élu ... De l'époque des balbutiements concurrentiels à celle du marché institué, les pompes funèbres n'ont guère eu recours aux techniques publicitaires ou de marketing classique, dès lors que celles-ci étaient assimilées à des signifiants ostentatoires du monde marchand. Condamnés à exister sur un marché qui doit se dénier comme tel, ils sont passés maîtres dans l'art de la dissimulation et de l'euphémisme dans la mise en scène de leurs pratiques. De fait, le jeu de la différenciation rencontre ici d'évidentes limites. Il est avant tout privé d'un certain nombre d'espaces publics où se déployer.

Les professionnels du funéraire partagent ainsi avec d'autres professions (avocats, médecins) la contrainte de non visibilité de l'offre, cependant pour des raisons très différentes. Publicité et démarchage commercial sont autorisés, à l'exception de celles réalisées à l'occasion ou en prévision d'obsèques. Plus encore, la question de la transparence sur les prix ${ }^{11}$, et plus largement de la définition d'un espace économique qui corresponde aux conditions du libre jeu de l'offre et de la demande ${ }^{12}$,

\footnotetext{
${ }^{11}$ Le décret du 9 mai 1995 relatif au règlement national des pompes funèbres définit les modalités d'information des familles et les obligations des régies et des entreprises ou associations habilitée à fournir des prestations funéraires. Il précise notamment les obligations concernant l'affichage de la liste des opérateurs funéraires habilités (mairie, établissements de soin et chambres funéraires), l'établissement d'un devis remis aux familles, les conditions générales associées au bon de commande.

${ }^{12}$ La normalisation des produits, services et équipements qui accompagne la loi de 1993 établit l'homogénéité des prestations de base sur l'ensemble du territoire national ; les règles strictes d'information du consommateur tentent d'organiser la transparence; la définition de «capacités professionnelles », assortie de contraintes de «moralité », pour les intervenants du secteur qualifie de façon uniforme les prestataires potentiels de ce nouvel espace marchand.
} 
ont fait l'objet d'investissements privilégiés en matière de réglementation, notamment dans le prolongement de la libéralisation du secteur (1993). Si les biens du marché funéraire sont «dotés de mystères », c'est donc moins parce qu'ils sont soustraits à l'évaluation directe d'une clientèle profane que par l'ostracisme que le public inflige à cette figure professionnelle inversée.

\subsection{Un client égaré}

La sanction sociale du marché ne pèse pas seulement sur l'entrepreneur, elle contrarie également fortement les dispositions consuméristes d'une clientèle peu encline à déambuler dans les boutiques de pompes funèbres dans l'éventualité d'un futur décès. De fait, l'achat de services funéraires est loin d'être systématiquement et raisonnablement anticipé, aussi bien par le futur trépassé que par ses proches.

Nos dispositions anthropologiques nous maintiennent confortablement à distance de la vie des morts, jusqu'au moment où nous sommes malheureusement rappelés à notre condition de simple mortels... L'inéluctable tabou de la mort, rappelle $\mathrm{P}$. Baudry (1995) après L. V. Thomas (1980), n'est que l'expression de notre humaine détermination à exclure la virtualité de notre disparition de notre vivant. «...Few people make plans for their own death, largely because of magical fears that to do so will hasten it. Most wills are drafted shortly before death (Dunham, 1963). Likewise, people rarely prearrange their own funerals despite the evidence that this reduces expenses considerably »(Zelizer, 1978). A distance du désir et de la jouissance consommatrice, l'achat de prestations funéraires est souvent dicté par l'impératif et la contrainte. Et quand bien même la situation échappe à la brutalité ou à la violence du décès accidentel, les proches éprouvent souvent quelques mauvais sentiments à quitter le chevet du mourant pour organiser ses obsèques avant l'heure.

Après d'intenses campagnes publicitaires, le développement récent du marché de la prévoyance en France est symptomatique de la montée de valeurs positives autour des contrats obsèques, à l'instar de ce qu'a montré $V$. Zelizer à propos de 
l'assurance-vie aux Etats-Unis ${ }^{13}$. L'entrée du troisième âge dans l'univers de l'assurance-décès n'en demeure pas moins modeste, notamment parce qu'elle se joue davantage sur le mode de la capitalisation que sur celui du règlement avant l'heure de ses propres funérailles (incluant la désignation d'un prestataire). On estime aujourd'hui que les contrats obsèques n'alimentent directement que $10 \% \mathrm{du}$ marché ${ }^{14}$. Le futur trépassé ne connaît donc qu'une influence limitée dans le processus de désignation d'un opérateur funéraire et d'organisation de ses obsèques. Et quand bien même il aurait pris soin de livrer ses desseins funèbres à ceux qui le célèbreront, il n'est pas rare qu'époux, enfants, parents du défunt accommodent ses dernières volontés à leur guise : en matière de funérailles, les auteurs principaux sont les vivants, non les morts. Ces derniers, même au cœur de la scène, sont paradoxalement absents...

A défaut d'être raisonnablement anticipé, l'achat de prestations funéraires sera réalisé sous contrainte de choc émotionnel et de temps. "Dans ces moments là... », évoquent interminablement les familles pour justifier leur défaillance. On aurait cependant tort de survaloriser le problème de l'entrave émotionnelle pour justifier la faiblesse du client. Toutes les familles ne sont pas éprouvées par le choc de la disparition plus ou moins brutale du parent affectionné. Au sein du collectif familial, la logistique des funérailles sera généralement prise en charge par les plus aguerris. La contrainte de temps pèse en revanche de façon systématique sur le processus de décision. La loi accorde un délai de six jours ouvrables pour l'inhumation ou la crémation. Mais la question de la prise en charge du corps défunt se pose de façon beaucoup plus immédiate, ne serait-ce qu'en raison de contraintes réglementaires (délai de transfert ${ }^{15}$ ). Appel nocturne, convocation en urgence par les soignants, découverte d'un corps au domicile : à l'annonce du décès, parents et proches sont pris dans l'impérieuse nécessité de composer avec l'être cher brutalement réduit à

\footnotetext{
${ }^{13}$ L'assurance décès est désormais considéré comme un moyen privilégié de libérer la descendance de la charge morale et financière des obsèques.

${ }_{14}$ «Les formules de prévoyance présentées par les professionnels funéraires ne représentent aujourd'hui que $10 \%$ du marché potentiel. En étant raisonnablement optimiste, on peut espérer voir les professionnels funéraires arriver à pénétrer $20 \%$ du marché. Au-delà, tout espoir est déraisonnable » (Funéraire magazine, $n^{\circ} 136$, Juin 2003).

${ }^{15}$ Les délais d'admission d'un corps avant mise en bière dans une chambre funéraire sont de $24 \mathrm{~h}$ à compter du décès et de $48 \mathrm{~h}$ lorsque le corps a subi des soins de conservation.
} 
l'état de dépouille. La crainte de l'entourage face au corps en décomposition et les prémisses d'un processus social et symbolique de détachement se caractérisent souvent par un empressement à mettre à distance le défunt. Dans l'enceinte des établissements de santé, principales demeures des défunts aujourd'hui, l'information du décès est souvent concomitante avec l'exigence d'une prise de décision immédiate ou rapide sur la destinée du défunt ${ }^{16}$ : transport en chambre funéraire, maintien au domicile, transfert à la morgue, soins thanatopraxiques.

La théorie économique considère le temps comme une variable fondamentale du processus d'achat. «Ce point est essentiel», nous indique C. Riveline (Riveline, 1991), « et il convient de s'y arrêter. En théorie économique, le décideur est résumé, avons-nous dit, par une fonction caractérisant ses préférences (...). Il est réputé nourrir ses calculs de toutes les informations nécessaires. Or en situation d'urgence, non seulement le nombre de données prises en compte par le décideur est limité par le temps dont il dispose pour s'informer, mais de plus, ses possibilités de recevoir des avis et de méditer sur son intérêt bien compris se trouvent réduites ».

Le manque d'anticipation ne saurait être compensé par la construction de l'expérience et la répétition de l'échange. La statistique nous donne l'achat de services funéraires comme une opération rare à l'échelle d'une vie ${ }^{17}$. Et même si elle ne reflète pas strictement notre taux de fréquentation des croque-morts ${ }^{18}$, on admettra aisément que le client est faiblement éclairé sur le contenu de la prestation. En dehors de la cérémonie proprement dite, il maîtrise mal les multiples exigences aussi bien techniques (maitrise de la thanatomorphose) que pratiques (organisation du service) qui constituent l'objet même du mandat confié à un prestataire.

\footnotetext{
${ }^{16}$ Le questionnaire médical du médecin en charge d'établir le certificat de décès amène à rapidement statuer sur le traitement du défunt : le corps peut-il être transporté ? Est-il apte à subir des soins thanatopraxiques ? Le médecin est censé répondre à ses questions uniquement en fonction de l'état médical du cadavre, mais il n'est pas rare qu'il s'entretienne avec la famille sur ce qu'elle envisage de faire du corps à cet effet.

${ }^{17}$ Une à deux fois en moyenne.

${ }^{18} \mathrm{Via}$ les enterrements notamment.
} 
Un achat plutôt exceptionnel (soit non répété), non anticipé et réalisé dans l'urgence, exposé au déficit de connaissances sur le contenu de la prestation, plus souvent motivé par la contrainte que par le désir : la conjugaison de ces différents facteurs nous livre une situation où le client est privé d'habiletés consuméristes et simultanément piégé dans les considérations pratiques et dans l'impératif de temps.

«Lorsque le deuil survient, l'exigence première est de permettre un "passage" digne au mourant, alors que le choix du prestataire doit s'effectuer rapidement, et que l'on ne connaît bien souvent aucune de ces entreprises. D'où, encore une fois, une forte ambivalence d'image des pompes funèbres : si, sur le moment, leur présence soulage - pris au dépourvu par le décès leur intervention apparaît comme réconfortante -, une fois passé le choc du deuil, le comportement rationnel de consommateur ressurgit : on a alors bien souvent le sentiment d'avoir été captif de l'entreprise. Non seulement le choix s'est effectué sans comparer prestations ni prix, mais nous nous sommes également aperçu que la plupart des personnes interrogées ne savent pas toujours précisément ce que recouvre la prestation » (Le vécu ou la perception du deuil et des obsèques. Etudes du CREDOC, 1999).

L'importance des investissements consacrés par les divers porte-parole du consommateur - média, presse consumériste et $\operatorname{DGCCRF}^{19}$ - à l'équipement de l'acheteur de services funéraires donne la mesure de sa faiblesse - ici désignée de vulnérabilité - qui plus est face à ces «marchands du temple» sur lesquels pèse immédiatement le soupçon d'arnaque. Depuis les années $80^{20}$, le consumérisme a trouvé en ce marché illégitime un terrain de prédilection. Reportages, dossiers, appels à la vigilance : le marché est perpétuellement dénoncé comme injuste. Mais l'éducation consumériste rencontre d'évidentes limites... Elle peut certes contribuer à légitimer l'expression d'un comportement calculateur - comparer des devis, consulter les prix par téléphone - dans un contexte où il est socialement considéré comme une infraction. Mais elle ne touche réellement qu'une fraction relativement minoritaire de la clientèle, celle qui accorde le primat au critère du prix pour une

\footnotetext{
${ }^{19}$ Direction Générale de la Concurrence, de la Consommation et de la Répression des Fraudes, administration publique en charge de la régulation des marchés.

${ }^{20}$ Les années 80 sont le théâtre de l'entrée en guerre de Michel Leclerc contre le monopole des PFG, alors détenteur de $80 \%$ du marché de la concession en France. Sa bataille s'appuie sur une forte médiatisation du conflit. Dans ce contexte, la presse consumériste et au-delà, l'ensemble des médias, ont porté un jugement sévère à l'égard de ceux que l'on dénonçait alors comme des marchands sans vergogne, profitant de l'ignorance et de la vulnérabilité des familles endeuillées.
} 
prestation qui, on l'a évoqué, motive généralement l'exigence de singularités liées à l'économie de la qualité.

\subsection{Du client égaré à la sociologie du marché}

Nous en savons maintenant assez sur les caractéristiques de l'acheteur de services funéraires pour nous intéresser maintenant à son devenir sur le marché concerné. Si l'on a pu évoquer des consommateurs à ontologies variables selon le degré d'anticipation de l'achat ou encore d'attachement à l'obligation d'hommage, domine néanmoins la rencontre avec un agent économique confronté à un achat impliquant et pour lequel se multiplient les entraves à l'élaboration d'un choix raisonné (émotion, urgence, ignorance). Cette figure sociale du «client égaré » n'est pas totalement inédite pour la sociologie du marché. On peut d'autant mieux l'affirmer que le modèle de «l'économie de la qualité » a été élaboré à partir d'une situation qui rencontre des contraintes similaires (Karpik, 1989). Le destin qu'on lui connaît jusqu'à présent est celui du secours d'intermédiaires propres à réduire l'incertitude sur la qualité et à soutenir sa quête d'orientation (Musselin, Paradeise, 2002). Si le «marché réseau » comme forme de médiation apparaît comme une modalité possible de la rencontre entre une famille et un opérateur funéraire ${ }^{21}$, nous faisons l'hypothèse que ce modèle de régulation n'est cependant pas dominant. Il est concurrencé par d'autres mécanismes, plus directement assimilables à la canalisation et l'aiguillage du client, comme alternative à un travail d'identification et d'évaluation d'offres différenciées.

On est donc immédiatement amené à questionner les motifs qui amènent notre client égaré à se soustraire de la recherche active d'information, ne serait-ce qu'a minima (bouche à oreille) pour au contraire se laisser conduire, guider, canaliser, tout au long

\footnotetext{
${ }^{21}$ Dans le cas qui nous occupe, il s'agit moins du réseau personnel (amis, connaissances) que d'interlocuteurs situés dans l'environnement immédiat du défunt. Les acteurs contemporains de la prise en charge du mourant - personnels soignants, membres de communautés religieuses ou culturelles, accompagnants associatifs - peuvent constituer des intermédiaires actifs pour les familles qui les côtoient durant l'attente plus ou moins longue qui précède le décès. Ceci étant, la médiation du réseau ne recouvre pas systématiquement une fonction de prescription, nous y reviendrons.
} 
du parcours qui va le mener à une boutique de pompes funèbres. Deux hypothèses explicatives peuvent être formulées. La première suppose que le consommateur, excessivement éprouvé par le dilemme entre primat de la qualité et obstacles à la rationalisation du choix, se trouve en quelque sorte en «état d'anomie » (Cusin, 2004) : «Le consommateur, conscient de ses limites cognitives et de la complexité de l'offre, risque d'être inhibé par l'angoisse de faire de mauvais choix. Bref, se laisser capter, c'est-à-dire accepter une restriction du champ des possibles et la délégation d'une partie de ses décisions, devient alors utile pour sortir d'une situation d'indétermination et d'incertitude... », ajoute l'auteur. Le client renoncerait alors à toute tentative élaborée de sélection au privilège du «choix qui s'impose », avec le bénéfice d'échapper à l'inconfort d'une décision aléatoire. La seconde hypothèse, que nous privilégions sans totalement déconsidérer la précédente, est celle de la puissance des dispositifs sociotechniques déployés par les entrepreneurs pour organiser son destin tracé. Dans ce scénario, tout se passe comme si «être actif ou réactif »- qualités sociologiques couramment attribuées au client engagé dans un processus complexe de choix - équivalait ici à «nager à contre-courant »... En d'autres termes, tout égaré soit-il, le client du marché funéraire n'évolue jamais dans un espace ouvert et indéterminé, mais il est au contraire très immédiatement amené à circuler dans un espace structuré.

Pour progresser dans l'analyse, il nous faut donc maintenant suivre ces clients acheminés malgré eux et les accompagner tout au long du processus séquentiel qui les conduit à mandater un opérateur funéraire pour l'organisation des funérailles. Nous suggérons de ne pas rester focalisés sur les tourments cognitifs de l'acheteur pour mettre davantage en scène l'efficace du process de service. Les professionnels du funéraire ont en effet acquis une grande familiarité avec les clients égarés qui peuplent leur marché. Ils ont appris à les repérer, parfois les dénicher. Ils ont développé des savoirs pour les appréhender sans se faire remarquer. Ils ont élaboré des équipements et des dispositifs de cadrage qui structurent, beaucoup mieux que le hasard, la rencontre marchande. Ce sont ces équipements matériels et symboliques de la captation que nous allons ici tenter de qualifier. Ils nous feront apparaître la face cachée de l'organisation du marché funéraire qui engage d'autres voies que celles de 
l'instrumentation de la qualification ou des réseaux sociaux. De ce point de vue, le secteur funéraire se révèle être un véritable laboratoire pour explorer ces modalités alternatives des dynamiques concurrentielles.

\section{L'organisation du process : réseaux professionnels et réseaux sociotechniques}

La littérature économique et sociologique a longuement exploré le caractère problématique de la transaction de service, parmi d'autres relations économiques ${ }^{22}$, du fait du différé dans le temps de la valeur d'usage, impliquant que le produit échangé ne préexiste pas à l'échange contractuel et intervient au contraire ex post. Mais il nous faut maintenant identifier toute une série de contextes où c'est au contraire la mise en œuvre d'une capacité sociotechnique, ne serait-ce que partielle, qui précède la contractualisation commerciale. Le déploiement du process ne constitue-t-il pas un moyen privilégié de cadrer la progression du client sur le marché et de «l'intéresser »- au sens de la sociologie des sciences et des techniques (Callon, 1986) - à une offre déterminée ?

Pour décrire cette autre modalité d'organisation de la relation marchande, nous suivrons donc les pompes funèbres dans le façonnage des réseaux sociotechniques qui structurent le process de service. Les procédés de conquête du client qui nous intéressent ont été, de tout temps, constitutifs des dynamiques concurrentielles au sein du marché funéraire. Les techniques et moyens mobilisés se sont transformés au cours de l'histoire, accompagnant les changements dans les modes de prise en charge du défunt, notamment avec la médicalisation de la fin de vie. Mais les principes qui gouvernent cette économie de la circulation demeurent relativement similaires d'une époque à l'autre, comme nous allons le voir.

\footnotetext{
${ }^{22}$ Cf. notamment les réflexions sur la relation de travail (Salais, 1989 ; Salais, Thévenot, 1986) ou la relation de sous-traitance (Baudry, 1994).
} 


\subsection{L'ordonnateur, le mandataire... et le bedeau}

La problématique de la captation surgit avec l'apparition de la concurrence à la périphérie du monopole des services funéraires, notamment dans les villes ${ }^{23}$. Les sociétés privées concessionnaires du monopole communal cherchent à asseoir l'exclusivité de leur marché face au développement des «agences de funérailles » qui leur disputent la vente des fournitures libres. Pour assurer leur commerce, ces dernières proposent au client de leur déléguer la négociation avec les sociétés concessionnaires de la ville pour l'organisation des funérailles ${ }^{24}$. Mais la capacité de ces mandataires à s'interposer entre les familles et les commis de la société de pompes funèbres de la ville n'a rien d'évident.

$\mathrm{Au}$ début du siècle, la mort à domicile ne permet pas encore de dessiner une cartographie de la mortalité. Le défunt est sédentaire: jusqu'aux obsèques proprement dites, il séjournera dans sa propre demeure, une fois celle-ci habillée par les pompes funèbres en «chambre mortuaire ». En revanche, la mairie est un point de passage obligé pour les proches, lieu alors hautement stratégique pour le règlement des affaires funéraires. La mairie est alors bien plus qu'une chambre d'enregistrement administratif des déclarations de décès, elle est le véritable siège du règlement commercial et logistique des convois. «L'ordonnateur», officier d'état civil $^{25}$ en charge de la planification des convois, et l'agent commercial de la société de pompes funèbres, y réceptionnent ensemble les familles pour l'organisation des funérailles. La connexion étroite entre ces différents acteurs ignore quasiment l'intromission possible d'intervenants concurrents. Déclaration d'état civil,

\footnotetext{
${ }^{23}$ La naissance des pompes funèbres profession de service associée à l'organisation des funérailles prend forme au $19^{\text {ème }}$ siècle, avec le fleurissement, au sein des grandes villes, d'entreprises spécialisées dans l'offre de prestations et fournitures nécessaires aux obsèques. Certaines ont le privilège d'acquérir la concession du monopole des convois et inhumations par les fabriques et les municipalités. D’autres, les «agences de funérailles», sont cantonnées dans le commerce des fournitures libres - fleurs, couronnes, etc.

${ }^{24}$ L'argument majeur mobilisé par les agences de funérailles est qu'elles préviennent les familles des abus potentiels des concessionnaires de la ville, du fait du privilège du monopole. Pour l'ensemble des acteurs du marché (concessionnaires, agences et fabriques), l'enjeu central est la composition du «panier de marchandises» sur la base du ratio entre fournitures non ou faiblement taxées et fournitures taxées (jusqu'à $50 \%$ de leur prix de vente) par les fabriques.

${ }^{25}$ Agent direct de la préfecture, employé de l'état civil et délégué du maire de l'arrondissement.
} 
organisation du convoi et achat de fournitures se déroulent de concert. C'est d'ailleurs bien l'intégration entre ces différentes composantes de l'organisation des funérailles qui établit la rencontre entre les familles et la société de pompes funèbres adjudicataire du service public comme hautement probable, sinon incontournable.

Les agences de funérailles, peu visibles $a$ priori, ne conquièrent leur place qu'à partir du moment où le client échappe à cette immédiate emprise du concessionnaire. D'où l'agitation fébrile aux portes des mairies rapportée par ce témoin de l'histoire, maire de la ville de Lyon.

"Les déclarations de décès se font dans six mairies d'arrondissement. $\mathrm{Au}$ voisinage de toutes les mairies, se sont ouverts des magasins de pompes funèbres. Les employés sont du matin au soir embusqués à la porte des mairies, à l'affût des gens venant déclarer un décès. Dès qu'un d'eux apparaît, il est entouré, racolé, pressé, tiraillé entre ceux qui se disputent sa clientèle » (Rubat du Merac, 1905).

On voit clairement ici les tentatives désespérées des mandataires pour précéder l'agent de pompes funèbres à l'entrée des mairies et ainsi contrarier le destin figé d'une famille, à l'origine essentiellement motivée par une déclaration d'état civil. Alternativement, l'agence de funérailles peut également tenter de partir à la chasse des familles endeuillées avant qu'elles n'atteignent la mairie. La boussole du commerçant le conduit alors vers tous ceux qui les côtoient avant l'heure, principalement les protagonistes du dernier sacrement...

\footnotetext{
"L'agence de funérailles n'attend pas le client ; il faut qu'elle aille à son devant : elle ne peut se contenter de « voir venir » le mort. Elle doit courir à la recherche du mourant. Mais oui ! C'est ainsi que les choses se passent. Un bon employé " d'agence " visite les églises, s'abouche avec " suisse ", " bedeau ", " sacristain ", «préposé aux chaises ", «donneur d'eau bénite » et toutes "gens d'église » informés qu'il suppose capables de le renseigner sur les clients de l'Eglise, l'ayant été... et susceptibles de le redevenir ». (Théodore-Vibert, 1905).
} 
L'agent qui réussit à briguer le mandat d'une famille n'est pas totalement au bout de ses peines. Son premier interlocuteur sera l'ordonnateur de la ville ${ }^{26}$, dont il doit obtenir les bonnes grâces pour s'assurer d'un «bon horaire» de convoi. En la matière, proximité et connivence avec l'adjudicataire de la ville bénéficient naturellement à ce dernier. «Ces commerçants indiscrets encombrent les bureaux de nos mairies, circonviennent nos employés pour obtenir pour les convois de leurs clients des heures de faveur », ajoute le maire de Lyon. Avec la disparition de l'ordonnateur comme figure d'intermédiation, les concessionnaires auront tout loisir de multiplier les obstacles afin de disqualifier l'intervention de l'agence de funérailles. Au sein de la régie ou du concessionnaire d'une grande ville, le responsable du planning des convois devient l'interlocuteur incontournable dont il est préférable de s'attirer les bonnes grâces, y compris par le biais de bakchichs.

\begin{abstract}
«Les familles sont régulièrement soumises à des pressions odieuses et parfois même intolérables. Dans certaines localités, les entreprises concessionnaires fixent en fait, selon leur bon vouloir, l'horaire des cortèges en prétextant, par exemple, l'impossibilité de fournir des porteurs ou le corbillard à d'autres heures. II va de soi que les familles qui ont accepté de confier le règlement de l'ensemble des funérailles, pompes extérieures et service intérieur, à la seule entreprise concessionnaire, ont toutes chance de bénéficier des horaires les plus convenables ». (Présentation de la proposition de loi sur les conseillers funéraires et les entreprises privées de pompes funèbres par Mme Troisier, 15 Janvier 1970).
\end{abstract}

Entre agences et concessionnaires, le jeu est inégal, mais cette asymétrie ne s'explique pas uniquement par les règles monopolistiques qui encadrent l'organisation économique de l'activité. Les uns, affiliés aux municipalités, tirent bénéfice de la confusion des espaces et des différentes modalités de règlement du convoi pour concentrer l'essentiel des flux de familles. Les autres, partisans d'une concurrence aux portes du monopole, se postent sur les itinéraires de passage des clients ou tentent d'enrôler tous ceux qui s'y trouvent « naturellement ».

\footnotetext{
${ }^{26}$ Jusqu'au début du $20^{\text {ème }}$ siècle, l'ordonnateur est un employé de la municipalité. Sa fonction disparaît ensuite pour être directement prise en charge par l'entreprise concessionnaire ou la régie municipale.
} 
De quoi nous parlent les stratagèmes des pompes funèbres en matière de conquête du client ? En premier lieu, d'intégration des activités à l'amont de la filière, assurant la confusion entre démarches administratives et négociation commerciale; en second lieu, d'alliances entre partenaires privilégiés, conjuguant réseaux publics, arrangements pratiques et bénéfices économiques.

\subsection{Des mairies aux hôpitaux}

Quelques décennies plus tard, au tournant du siècle (1960), nous retrouvons notre escouade de croque-morts concurrents en proie à la même agitation, mais transportée en d'autres lieux. Les boutiques de pompes funèbres quadrillent les environs immédiats des hôpitaux, hauts lieux de concentration de la mortalité. Les dispositions sont sensiblement les mêmes, à ceci près que l'on ne canalise plus seulement les familles, mais les défunts eux-mêmes. Plutôt que de se poster sur les itinéraires de passage des familles endeuillées, les entreprises de pompes funèbres partent à la conquête des cadavres.

Avec la médicalisation de la fin de $v^{2}{ }^{27}$, les établissements de soin concentrent des flux de défunts hier dispersés. Dépassés par cette soudaine invasion mortuaire dans leur enceinte, ils ne sont encore pas prêts à recueillir des dépouilles encombrantes sur le plan pratique comme sur le plan symbolique. Les opérateurs privés rencontrent ici une opportunité providentielle de conquérir «l'amont de la filière ». Ils investissent dans un nouvel équipement, les «chambres funéraires », dépôts mortuaires dont ils assurent la gestion complète. L'offre est immédiate, efficace et moins coûteuse, elle dispense provisoirement les établissements de santé d'investir dans des équipements ad hoc. A partir du début des années 60, on assiste ainsi au développement des « chambres funéraires » qui se substituent aux morgues hospitalières publiques pour devenir le lieu privilégié d'accueil des cadavres. Sur le modèle des funeral-home américain, la chambre funéraire, autrement nommée « funérarium », est une morgue

\footnotetext{
${ }^{27}$ On peut rappeler l'ampleur de ce mouvement de médicalisation de la mort. En 1964, on compte encore $63,2 \%$ de décès au domicile contre 33,5\% en établissement hospitalier (hôpital, hospice, maison de retraite). Vingt ans plus tard, ce rapport s'est inversé. En 1983, au plan national, 66\% des décès ont eu lieu en établissement hospitalier et seulement 30,5\% au domicile.
} 
privée, gérée par une entreprise de pompes funèbres, dans laquelle peuvent être déposés, conservés, apprêtés et veillés les défunts. Echoués aux portes des hôpitaux et pas encore apprêtés pour les funérailles, les défunts disposent ainsi d'un lieu de séjour provisoire qui leur est entièrement consacré : en «back office », conservation au sein de locaux techniques (casiers réfrigérés), salle de soin (toilette, thanatopraxie); en «front office », local d'accueil, salons de présentation du corps, salle de cérémonie.

On pourrait analyser la chambre funéraire comme une innovation en terme d'offre de service qui participe d'une considérable transformation et amélioration de la qualité de service. Mais pour les pompes funèbres, elle est aussi et surtout un moyen de maîtriser plus directement la circulation des défunts. Depuis les années 70 jusqu'à nos jours, la cartographie du commerce funéraire s'est ainsi dessinée pour une large part à partir des chambres funéraires. Les PFG, leader du marché privé sous l'ère du monopole communal, doivent à ce dispositif anglo-saxon dont ils ont la paternité en France leurs années de gloire $(70-80)^{28}$. En l'espace d'une vingtaine d'années, près de 200 établissements sont implantés sur le territoire français, principalement à l'initiative du groupe. Adossant le monopole de la concession de service à celui de la gestion de chambres funéraires ${ }^{29}$, les PFG gouvernent durablement l'activité au sein des centres urbains.

\footnotetext{
${ }^{28}$ L'entreprise des Pompes Funèbres Générales a grandi tout au long du $20{ }^{\text {ème }}$ siècle sur la base du développement et de la conquête du marché de la concession, qu'il détient à 80\% (2900 communes) jusqu'à la libéralisation. A côté de ce leader d'envergure nationale (environ 1500 agences réparties sur l'ensemble du territoire), la structure de la concurrence est atomisée. "Au niveau national, les obsèques sont assurées pour $41 \%$ par une seule grande entreprise et ses filiales, pour $46 \%$ par de nombreuses autres entreprises indépendantes » (Barrau, 1987).

${ }^{29}$ Cf. Rapport sur l'organisation du service des Pompes funèbres, IGF-IGA-DGAS, 1989. Plusieurs motifs concourent à cette convergence : d'une part, les concessionnaires (notamment les PFG) sont les seuls à pouvoir supporter de tels investissements, chiffrés à plusieurs millions de francs selon l'importance et le confort des équipements. En contrepartie, la prorogation de la concession par les élus locaux vient naturellement justifier les immobilisations consenties par le concessionnaire, dans la mesure où ces équipements mortuaires viennent très souvent pallier le déficit des établissements publics de santé dans la gestion de la sortie de vie.
} 


\subsection{La chambre funéraire comme plate-forme stratégique}

Avec la chambre funéraire, on peut dire que les tactiques de rencontre ont gagné en subtilité, restaurant ainsi la discrétion dont les pompes funèbres sont coutumiers... Le bricolage artisanal du siècle précédent fait place à des équipements beaucoup plus élaborés : les leaders du secteur ne se contentent plus de se poster sur les itinéraires probables des familles endeuillées, ils organisent la circulation des dépouilles.

Jusqu'à la fin des années 80, l'absence de stricte réglementation en la matière, l'engouement des hôpitaux pour des solutions externes susceptibles de pallier rapidement le déficit d'équipement, l'intérêt des entreprises de pompes funèbres à se rapprocher de ces lieux de concentration de la mortalité, encouragent largement la substitution des morgues privés à celles des établissements publics de soins. Au cours des premières décennies d'apparition des chambres funéraires, leurs gestionnaires privés bénéficient le plus souvent de conventions avec les directions d'établissements de soin qui font du funérarium leur morgue, ce qui les dispense d'en construire et de les gérer ${ }^{30}$. Il n'est d'ailleurs pas rare que l'opérateur funéraire encourage cette délégation en offrant la gratuité des frais de transport et de séjour, ce qui évite de surcroît toute controverse sur la substitution d'une offre privée à l'offre publique $^{31}$. Le chaînage étroit entre équipement médical et équipement mortuaire passe également par la proximité géographique, voire l'intégration directe: les pionniers du développement des chambres funéraires n'hésitent pas, quand ils le peuvent, à bâtir celles-ci au sein même de l'enceinte de l'hôpital. A défaut, elles se logent à proximité immédiate de la sortie de l'établissement, situation qui se généralise à partir de 1993 sur la base des décisions de justice et textes normatifs qui imposent la séparation physique des locaux. Ainsi annexée à l'établissement de

\footnotetext{
30 «Ni les hôpitaux publics, ni les établissements privés ne sont incités au développement rationnel des chambres mortuaires dans la mesure où une disposition réglementaire autorise les directeurs d'établissements à se substituer à la famille dans le cas où elle ne peut être jointe et à faire transférer dans une chambre funéraire le corps d'une personne décédée dans un établissement » (Rapport sur l'organisation du service des Pompes funèbres, IGF-IGA-DGAS, 1989).

31 La prestation assurée par le funérarium est rémunérée, contrairement à celle des morgues hospitalières publiques. Pour prévenir un usage abusif de la chambre funéraire par les directions des établissements de soin, la réglementation établit que toute demande d'accueil d'un défunt à l'initiative des directions sera à la charge de l'établissement.
} 
santé, la chambre funéraire participe immédiatement au process de traitement des cadavres. La conservation en casier réfrigéré s'établit pratiquement et symboliquement comme une étape supplémentaire de la chaîne du soin ou du «post soin ». A défaut d'unité en propre consacrée à cet effet, les personnels soignants n'hésitent pas à contacter directement les opérateurs funéraires pour l'évacuation d'un corps. Les employés funéraires évoluent librement au sein de l'hôpital pour la levée du corps et se substituent au soignant pour la toilette mortuaire, les soulageant totalement de la prise en charge du défunt.

En immédiate proximité avec les lieux de concentration de la mortalité, les chambres funéraires constituent de véritables «autoroutes»drainant les cadavres que les hôpitaux se pressent d'évacuer. Les entreprises acquièrent ainsi les moyens de faire venir à elles les clients. En absorbant les flux de cadavres dont les établissements de soin sont submergés, elles canalisent les proches qui transitent automatiquement par ce lieu de dépôt du corps. Ensuite, il ne reste plus à l'opérateur funéraire qu'à précéder la famille pour passer de la chambre funéraire au conseiller commercial réglant les obsèques.

L'efficacité de tels équipements en matière de captation est indéniable: le déséquilibre dans la distribution du marché est à la source de nombreux litiges et recours judiciaires entre opérateurs funéraires ${ }^{32}$. Il motive de successives dispositions réglementaires destinées à rétablir la compatibilité entre la gestion des chambres funéraires et la liberté concurrentielle des funérailles (Auby, 1997) ${ }^{33}$. Dès 1987, la réglementation prévoit notamment le libre accès des agences de funérailles aux chambres funéraires. Il faut attendre la loi de 1993 et les successifs textes d'application qui l'accompagnent ${ }^{34}$ pour que les dispositions réglementaires concernant la chambre funéraire imposent une stricte distinction entre les chambres

\footnotetext{
${ }^{32}$ On s'appuie ici notamment sur l'étude systématique des décisions du Conseil de la Concurrence concernant les recours des opérateurs funéraires devant la DGCCRF.

${ }^{33}$ Les dispositions du décret du 14 janvier 1987 (puis de la loi du 8 janvier 1993) ont abouti à désigner les chambres funéraires comme des équipements d'utilité publique, exerçant une activité de service public, et susceptibles d'être gérées concurremment par toute régie, entreprise privée, association, sous réserve qu'elles aient été habilitées (Auby, 1997).

${ }^{34}{ }^{\text {Décret }} \mathrm{n}^{\circ}$ 94-1027 du 23 Novembre 1994 ; Décret nº 97-1039 du 14 novembre 1997 ; Décret nº5653 du 9 mai 1995 relatif au règlement national des pompes funèbres.
} 
mortuaires (morgue hospitalière) et les chambres funéraires privées. Il n'en reste pas moins que la faculté d'absorption des cadavres qu'apporte la chambre funéraire bénéficie essentiellement à ceux qui en assurent la gestion déléguée, c'est-à-dire aux opérateurs qui, après autorisation des autorités publiques ${ }^{35}$, ont consenti à cet investissement coûteux ${ }^{36}$, avec le privilège de ne rencontrer aucune concurrence directe sur le périmètre territorial couvert.

\subsection{Les « barons locaux »}

D'un territoire à l'autre, la structure de la concurrence découle très largement de la «distribution des ressources» au sein de la filière de traitement des défunts. La chambre funéraire forme un puissant levier de constitution d'un monopole économique de fait, situation qui vaut par le passé (monopole communal) comme par le présent (concurrence libre).

Jusqu'à la libéralisation, la gestion de la chambre funéraire démultiplie la position dominante du concessionnaire vis-à-vis des agences de funérailles. Par son action supplétive à celle des pouvoirs publics, elle contribue à le conforter comme partenaire privilégié des collectivités locales. La puissance des «barons locaux » conjugue alors deux ressorts étroitement liés : d'une part, l'intégration dans la chaîne des soins, via l'agencement des équipements et l'affiliation des univers professionnels de la santé et de la mort; d'autre part, la complicité des multiples protagonistes issus des pouvoirs publics intervenant directement dans le traitement des défunts. Les relations étroites entre opérateurs funéraires, établissements de soin et pouvoirs publics se prolongent en effet par des liens de proximité et d'interconnaissance étroits noués dans le cadre de l'activité funéraire. Les soignants, la police (contrôle et traçabilité du cadavre), les employés publics (mairie, cimetière),

\footnotetext{
${ }^{35}$ Une chambre funéraire constitue un équipement dont l'utilité publique doit être établie par les autorités municipales et préfectorales. Ce contexte administratif et juridique limite étroitement l'implantation d'équipements concurrents au sein d'un même territoire. Il s'accompagne de stratégies de lobbying politique visant à empêcher l'implantation de chambres concurrentes sur un périmètre territorial donné.

${ }^{36}$ Actif d'une valeur généralement comprise entre 0,1 à 0,4 millions d'euro, il suppose une capacité d'investissement à ne pas minimiser.
} 
constituent des intermédiaires essentiels susceptibles d'acheminer défunts et familles vers un prestataire désigné. Directement en prise avec ces réseaux publics, les concessionnaires et régies bénéficient ici d'un net avantage sur leurs concurrents en agence. En la matière, il n'est d'ailleurs pas totalement exclu que les échanges interprofessionnels aient été parfois soutenus par des rémunérations occultes.

«Il résulte de l'enquête administrative qu'une certaine orientation de la clientèle vers les $\mathrm{PF}^{* * *}$ peut être organisée au moyen du versement de gratifications à des personnels de mairie (état civil, services techniques), aux conservateurs de cimetière ou des fossoyeurs, à des secrétaires de mairie, à des personnels hospitaliers. Le rapport d'enquête relève que celles-ci sont parfois la contrepartie de servies effectivement rendus (service administratifs, toilettage, habillage de la personne décédée...) » (Rapport de M. PAULOT relatif à la position dominante du groupe des $\mathrm{PF}^{* * *}$ pour la Cour d'Appel de Paris (1986).

L'alliance entre ces différents protagonistes privés et publics du process de traitement des défunts trouve cependant bien d'autres motifs que les bakchichs ou avantages en tout genre ${ }^{37}$. «Affiliation commune ${ }^{38}$ et arrangements coopératifs constituent des ressorts largement suffisants, nous y reviendrons. Au contraire, les phénomènes de corruption les plus répandus, essentiellement durant les années 80 , ont davantage été localisés au niveau des morgues hospitalières parisiennes, sur un territoire où les chambres funéraire sont singulièrement rares. De fait, en l'absence d'équipement de substitution dans la prise en charge de la mortalité hospitalière, la morgue redevient un espace central d'interface avec le marché où se négocie âprement la distribution des défunts et de leurs proches. D'autant que la ghettoïsation des morgues au sein des établissements de santé est propre à produire une population particulièrement disposée à recevoir des compensations monétaires...

\footnotetext{
${ }^{37}$ L'un des derniers BO du Conseil de la Concurrence relatif aux pratiques mises en œuvre sur le marché des pompes funèbres évoque dans ses constatations, la distribution de cadeaux de fin d'année par l'opérateur dominant à destination des cadres et directeurs de maison de retraite, de mutuelles, de la police et de la gendarmerie, des maires, des soignants du CHR (surveillante, infirmière et autres personnels). Ces pratiques ont été considérées comme « des pratiques commerciales courantes ».

${ }^{38}$ L'appartenance des agents de pompes funèbres aux réseaux publics vaut pour les concessionnaires privés du temps du monopole, mais également et de tout temps pour les régies et Société d'Economie Mixte.
} 
«Le secteur funéraire est un marché économique local: le marché des services funéraires reste, nonobstant son ouverture totale depuis l'abrogation du monopole communal des pompes funèbres, un marché local qui est largement structuré par la localisation du mort, en général l'hôpital ou la chambre funéraire » (d'Abbadie, 2003). La construction des réseaux sociotechniques concentre les stratégies concurrentielles, reléguant au second plan la concurrence sur les prix et les qualités. Les disparités importantes de tarifs au sein d'un même territoire local attestent de l'absence de concurrence par les prix ${ }^{39}$, également confortée par l'opacité entretenue par les prestataires dans l'équivalence prestation/prix ${ }^{40}$. Par ailleurs, on trouverait ici une clé de compréhension des limites aux effets attendus de la libéralisation. Les réformateurs du monopole communal ont confié à la concurrence libre les vertus d'un «assainissement du marché », au bénéfice d'une démocratisation des obsèques (baisse des prix). Pour garantir le succès d'une telle entreprise, ils ont assorti la levée du monopole d'une multiplication des dispositions en faveur de la transparence et de l'équipement consumériste du consommateur ${ }^{41}$. Placé sous surveillance des pouvoirs publics, l'institution pionnière du «service public concurrentiel» des pompes funèbres n'a cependant pas apporté les résultats escomptés. Depuis 1993, on assiste au contraire à une inflation galopante des prix, bien supérieure à celle de l'indice d'ensemble des prix à la consommation ${ }^{42}$. Le constat sur le terrain est double : d'un côté, les PFG ont maintenu une politique de prix élevés, jusqu'à $30 \%$ supérieur à

\footnotetext{
39 L'étude réalisée en 1997 par le CRC-Consommation sur la région Nord-Pas de Calais, à partir de 1062 relevés de plus de 70 entreprises, établit nettement l'importance des disparités en matière de prix. On relève notamment que : - Les écarts de prix entre deux zones s'établissent dans une fourchette comprise entre 0,7 et $36,1 \%$; En considérant les écarts entre zones selon le produit, on constate un écart très important des prix des fournitures annexes telles que les faire-part, la toilette du défunt, l'urne de base ; le prix d'une zone à l'autre pouvant être multiplié par 5; - L'analyse par type de produit ou de prestation montre que quel que soit le produit considéré, les fourchettes de prix sont très importantes.

${ }^{40}$ Les différences de terminologie, les forfaits regroupant plusieurs prestations, les différences dans la prise en compte des services connexes (frais de tiers, marbrerie) rendent les devis souvent difficilement comparables.

${ }^{41}$ Décret $n^{\circ} 95-653$ du 9 mai 1995 relatif au règlement national des pompes funèbres.

42 «L'analyse permet de dégager les tendances suivantes : - sur la période 1998-2002, les prix des prestations funéraires ont augmenté de 11,4\% soit moins que sur la période 1992-1997 ; - Après un infléchissement du rythme de hausse des prix en 1999 et 2000, ces dernières se sont accélérées depuis 2001. En évolution annuelle, les prix des services funéraires ont augmenté de $+2,5 \%$ en $1999,+2,2 \%$ en $2000,+2,9 \%$ en $2001,+3,2 \%$ en 2002 . L'évolution de l'indice des prix des prestations funéraires demeure très supérieure à celle de l'indice d'ensemble des prix à la consommation hors tabac » (Rapport du Conseil National des Opérations Funéraires, 1998-2002).
} 
ceux de leurs concurrents dans certaines localités. De l'autre, les PME se sont dotées de chambres funéraires, au prix de lourds investissements à l'échelle de ces petites structures.

Cette lecture des dynamiques concurrentielles permet en revanche de comprendre l'intensité des débats et négociations entre Etat et fédérations professionnelles concernant les dispositions législatives de 1999 qui imposent la réhabilitation des chambres mortuaires au sein des établissements hospitaliers ${ }^{43}$. Une telle disposition participe directement au déploiement d'une alternative publique à la chambre funéraire privée. La chambre mortuaire hospitalière offre une gamme de service à peu près équivalente à celle de la chambre funéraire, avec cependant une qualité de service très inférieure ${ }^{44}$. Elle n'en satisfait pas moins les petits opérateurs qui disposent de cet équipement public pour l'ensemble des soins au défunt, avec un moindre coût du service final compte tenu de la gratuité de l'hébergement. En modifiant le process de traitement du défunt, la chambre mortuaire freine la canalisation des corps par les chambres funéraires privées. Elle constitue un équipement concurrent à l'amont de la distribution du marché, contribuant à réduire la position dominante des leaders du marché local face aux petites agences concurrentes. Provisoirement, peut être... Objet d'intenses pressions politiques, l'avenir des chambres mortuaires est plus qu'incertain...

\footnotetext{
${ }^{43}$ En 1999, le législateur établit par décret l'obligation pour tout établissement de santé accueillant plus de 300 décès par an de disposer d'une chambre mortuaire aux normes ainsi que d'un service mortuaire hospitalier pour l'accueil des familles.

44 Décor sobre, atmosphère hospitalière, faible disponibilité des intervenants, horaires d'accueil limités. La gratuité de l'hébergement du corps est limitée à trois jours.
} 


\section{La « fluidité industrielle ${ }^{45}$ » au cœur du marché}

Une fois reconnue la puissance de cette modalité de régulation du marché, il nous incombe de mieux qualifier les ressorts de l'activité de captation en tant que telle. Nous avons jusqu'à présent cartographié les rivières et chenaux où circulent les flux de défunts, ces intermédiaires essentiels par lesquels peut s'établir la relation entre clients et opérateurs funéraires. Il nous faut également repérer les procédés de signalisation, les techniques d'adressage, les processus décisionnels associés à l'acheminement des corps en amont de la filière, bref l'ensemble des dispositifs qui participent à la canalisation du cadavre et/ou à la structuration de la rencontre entre la famille et l'opérateur.

Ces dispositifs nous sont notamment rendus visibles par la contestation des agences de funérailles qui espèrent contrarier ce destin figé du client pour accéder à une visibilité et commencer à exister sur le marché. Outre le corpus classique d'enquête de terrain, l'analyse des conflits portés à l'arbitrage de la DGCCRF livre un matériau précieux témoignant des multiples stratagèmes déployés par les opérateurs dans leur entreprise de conquête des défunts ${ }^{46}$. Ces controverses et leur dénouement sont riches d'enseignement pour alimenter notre connaissance de la variété des équipements et dispositifs participant à l'organisation de la trajectoire du client au fil de la régulation du process. Nous observerons de quelle façon la discontinuité pratique des espaces sociaux s'efface au bénéfice d'un cheminement fluide et continu menant

\footnotetext{
${ }^{45}$ J'emprunte cette notion à F. Vatin (Vatin, 1987) qui l'a élaborée à partir de l'analyse des formes d'organisation industrielle associées aux industries de process. Elle met en évidence la centralité de la continuité productive et de la fluidité technique comme principe organisateur des systèmes productifs. G. Rot a particulièrement bien montré la portée heuristique de ce concept dans l'analyse de dispositifs organisationnels au sein de contextes industriels a priori éloignés des industries de flux (Rot, 2002).

Bien que transportant le concept dans le champ des services, il nous semble pertinent de maintenir le qualificatif «industriel » en l'identifiant davantage au mouvement de standardisation des processus qu'à un secteur économique déterminé (Segrestin, 1997).

${ }^{46}$ Les citations sont le plus souvent extraites de la partie préliminaire restituant le constat des faits, indépendamment de l'avis de la DGCCRF. Ils témoignent donc des récriminations des agences à l'encontre des gestionnaires de chambre funéraire mais pas nécessairement d'une condamnation par le Conseil de la Concurrence.
} 
inexorablement à un opérateur funéraire. $\mathrm{Si}$, pour les morts comme pour les vivants, la vie est un long fleuve tranquille, c'est essentiellement grâce aux réseaux sociotechniques (Latour, 1989 ; Vinck, 1999) qui fabriquent le maillage des espaces et le continuum des actions entre les différents lieux où circulent le défunt et ses proches.

\subsection{L'amont de la filière : organiser la « commutation automatique »}

Naissance de la commutation automatique
«Dans les centraux manuels, l'opératrice pratique directement la connexion entre l'abonné et son
correspondant à l'aide de jacks ou fiches. Dès la fin du XIXe siècle, on invente la commutation
automatique et semi-automatique. C 'est à Kansas City qu'un entrepreneur des pompes funèbres,
Almon B. Strowger, soupçonnant la demoiselle du téléphone local de renvoyer tous les appels vers
son concurrent, met au point, en 1891, un système de connexion automatique au central. Cinq ans plus
tard, le cadran téléphonique, permettant à l'abonné de demander directement son numéro, est inventé.
"France Télécom, Mémoires pour l'action", 1995, p. 56.

L'affaire de Kansas City n'est pas sans intérêt pour l'histoire du téléphone, mais elle ne l'est pas moins pour nos affaires de croque-morts. Car cette jeune femme dévouée à la cause de son époux, entrepreneur de services funéraires, ne fait autre chose que d'établir astucieusement la rencontre première entre l'offre et la demande sur le marché des services funéraires.

De diverses façons, les opérateurs funéraires tentent parfois eux aussi de réinventer la « commutation automatique » avec les principales scènes où surviennent les décès : l'établissement de soin (hôpital, clinique, maison de retraite) ou le domicile du défunt. C'est ainsi que le leader local d'une grande agglomération urbaine a réussi à établir sa ligne téléphonique comme numéro interne de l'un des principaux établissements de soin du lieu. Le maillage téléphonique peut également s'étendre aux divers intermédiaires publics susceptibles d'entrer en contact avec les familles endeuillées.

\footnotetext{
${ }^{47}$ Nous remercions vivement Laurent Menegoz de nous avoir communiqué cet extrait.
} 
« II a été établi que 5 communes sur les 55 adhérentes à la SAEM-PF*** communiquaient les seules coordonnées de la SAEM-PF*** à leurs administrés en cas de décès (message répondeur ou affichage en mairie). Par ailleurs la SAEM-PF ${ }^{* * *}$ a bénéficié, jusqu'en juillet 2000 , d'une liaison téléphonique directe avec la mairie de $X^{* * *}$ qui abrite le bureau des cimetières et le bureau de l'état civil " (BOCCRF $n$ ²004-09)

Plus répandus sont les multiples procédés de signalisation tels que les en-têtes d'entreprises privées apposées sur des formulaires administratifs obligatoires ${ }^{48}$.

«La première note d'information diffusée par l'hôpital rappelait la liberté de choix des familles pour le transfert du corps de leur défunt, mais ne mentionnait que le nom du funérarium de $X^{\star \star *}$ et les conséquences financières du choix de cette destination. La note diffusée depuis le 25 janvier 1994 fournit la liste des principales chambres funéraires de la région de $P^{* * *}$. Le formulaire de demande de transport de corps avant mise en bière utilisé par l'hôpital jusqu'au dernier trimestre 94 comportait la mention imprimée: "demande de transport au funérarium de la société des Pompes Funèbres $X^{\star \star \star} »$ (extrait de l'instruction rapporté par le BO de la DGCCRF, n8 du mardi 29 avril 1997 - souligné par nous)

Pour ces proies totalement imprévisibles que sont les défunts à domicile, les numéros verts «SOS décès » apposés dans les pages publicitaires des revues du troisième âge reproduisent sensiblement cette technique d'adressage téléphonique inventée par la « demoiselle du téléphone ». L'appel au «SOS décès » est directement réceptionné par les commerciaux des agences funéraires.

\subsection{Agencements et arrangements}

Pour la plupart de nos contemporains défunts, on l'a évoqué, la structuration des itinéraires de circulation des clients repose en premier lieu sur cette foule d'acteurs professionnels qui gravitent autour d'eux et en décident l'orientation : le médecin qui

\footnotetext{
48 Il s'agit notamment des demandes d'admission en chambre funéraire ou des demandes d'autorisations de transport avant mise en bière, qui doivent être signées par la famille. Ces autorisations sont obligatoires pour tout transport du défunt au sein d'une chambre funéraire ou en dehors de la commune. Il s'agit de documents juridiques produits par l'administration et transmis par les soignants ou les employés funéraires à la famille.
} 
atteste du dernier soupir, le policier qui ordonne une réquisition ${ }^{49}$, l'ambulancier qui transporte le moribond, l'infirmière qui administre l'occupation d'un service clinique, le garçon de morgue qui accueille les défunts du lieu.

« II est établi que contrairement à la réglementation applicable, le directeur de la maison de retraite $\mathrm{B}^{\star \star \star}$ faisait signer aux familles, lors de l'admission de l'un de leurs membres, une demande d'admission au funérarium. En outre, le gérant de cette maison de retraite faisait exclusivement appel à l'ambulancier de l'entreprise $X^{* * *}$ » $(B O$ de la concurrence, de la consommation et de la répression des fraudes $-n ³$ vendredi 2 février 1990).

L'intervention de ces professionnels est centrale dans la distribution du marché. Elle peut classiquement se situer sur le registre de la «prescription », soit la transmission d'informations et de savoirs participant au processus de qualification du service ou du prestataire $\left(\right.$ Hatchuel, 1995) ${ }^{50}$. Elle peut, à l'autre pôle, participer à des relations d'ententes voire de corruptions malheureusement longtemps dénoncées dans ce secteur. Mais elle peut aussi, dans nombre de cas, s'organiser au regard de contraintes déterminantes en matière de régulation du process et de gestion des flux. C'est à ce titre qu'elle intéresse plus directement la problématique de la captation, en constituant l'un des lieux investis par les opérateurs de services funéraires pour organiser les itinéraires de circulation du défunt.

«Par courrier en date du 18 octobre 1999 adressé au directeur du
CHRU, le directeur des PF** attirait l'attention de celui-ci sur
l'orientation automatique du corps de défunts, par le personnel de
l'hôpital, vers la chambre funéraire des PF $\diamond$ [gestionnaire] (...cite le cas
de plusieurs familles victimes de ces pratiques..). Les $\mathrm{PF}^{\star * *}$ rapportent
aussi le cas de M. [identité] dont la famille a demandé le transfert du
corps à la chambre mortuaire du CHRU. Les infirmières ont conseillé à
cette famille de faire appel aux PF $\diamond:$ «il faut faire appel aux PF $\diamond$ pour le
transfert au centre funéraire, c'est beaucoup plus rapide ici on fait
toujours comme ça » (BOCCRF n`2004-9).

\footnotetext{
${ }^{49}$ En cas de découverte d'un cadavre, si la cause de la mort est inconnue ou suspecte, c'est l'officier de police judiciaire qui intervient sur les lieux et réquisitionne une entreprise de pompes funèbres pour le transport du corps en chambre mortuaire ou funéraire.

${ }^{50}$ L'auteur apparente le processus d'achat d'un bien ou d'une prestation à l'expression d'une délibération et d'un jugement sur trois éléments : la chose ou la prestation acquise, son mode de jouissance ou d'usage, l'appréciation qui sera portée sur celles-ci. La figure du "prescripteur" caractérise le recours à un tiers pour l'acquisition d'informations et de savoirs concernant ces différents éléments.
} 
Libérer l'espace, dégager les morts, récupérer des lits pour l'accueil des malades, telle est l'affaire de ces professionnels qui rencontrent ponctuellement ou quotidiennement des cadavres sur leur chemin. Au sein des établissements de santé, la pression sur la disponibilité des lits ne cesse de s'accroître. Il est courant que l'infirmière cadre ou la direction de la maison de retraite, soucieux de l'évacuation rapide du défunt, n'attendent pas le mandat de la famille pour solliciter directement l'intervention d'un opérateur funéraire. En d'autres termes, pour les soignants comme pour le gendarme ou le pompier, évacuer un corps de la chambre hospitalière ou de la voie publique relève d'une contrainte propre et d'une responsabilité professionnelle avant d'être l'affaire de la famille. La rapidité d'intervention, les facilités de coordination et autre forme de complicité de tous ordres participent directement aux critères de choix d'un opérateur funéraire par ces professionnels : s'il y a processus de qualification, celui-ci se construit à partir du monde professionnel de l'intermédiaire, non de celui du client.

Là encore, l'opérateur funéraire gestionnaire d'une chambre funéraire dispose d'atouts considérables vis-à-vis de ses concurrents pour répondre plus efficacement aux contraintes de coordination et de logistique des transports de corps. Le transport d'un corps d'une commune à une autre est soumis à l'obtention d'autorisations administratives associées à une traçabilité policière rigoureuse ${ }^{51}$. Le process est donc constamment systématiquement suspendu à l'intervention sur place de l'agent public ayant pouvoir de police pour la délivrance des autorisations (maire, garde-champêtre, policier). Or le principal poste de contrôle des autorisations se situe précisément à l'entrée de la chambre funéraire. Il est donc beaucoup plus aisé pour le gestionnaire de la chambre de se dispenser temporairement des obligations administratives.

«Pour aller chercher un corps à la clinique $A^{* * *}$, on doit d'abord aller chercher les papiers à la clinique, laisser le corps mais prendre les

\footnotetext{
51 Les opérations funéraires sont soumises à des contraintes réglementaires et des contrôles policiers qui impliquent la présence d'agents de l'état sur les lieux (policier, maire), notamment pour le transport d'une commune à une autre, puis ultérieurement la fermeture du cercueil et l'inhumation ou la crémation. Cette présence obligatoire implique d'importantes contraintes de coordination.
} 
papiers, les trois papiers signés par le médecin et la famille ${ }^{52}$, retourner au bureau (police), qu'il nous fasse l'autorisation de transport avant mise en bière et qu'on retourne chercher le corps une fois que le document est tamponné par le flic. Déjà, il y en a pour $3 / 4$ d'heures et vous n'avez toujours pas ramené le corps. Et après seulement vous ramenez votre corps à la chambre funéraire (CF). Les $\mathrm{PF}^{* * *}$ (gestionnaire de la CF), comment voulez-vous qu'ils fassent cela, ils en font six ou sept par jour... Ils font six civières, il y aurait une équipe pour ne faire que les civières. Donc eux, ils vont chercher directement les papiers, le corps, ils font l'admission [en CF] tranquillement et en fin de journée, ils amènent tout au bureau (police) et l'autre régularise : " tu l'as amené quand, à quelle heure... ». Nous, c'est impossible de faire cela, nous, il faut qu'on fasse tout le circuit, on met quatre fois plus de temps pour aller chercher un corps, ce n'est pas normal ».

$\mathrm{Au}$ policier de garde qui doit abandonner un repas dominical en famille pour la «pose d'un bracelet ${ }^{53}$, au maire du village contraint de se déplacer en pleine nuit au domicile du défunt, à la surveillante du service clinique qui attend la famille pour obtenir son mandat, le gestionnaire peut offrir la possibilité d'une régularisation $a$ posteriori des procédures réglementaires.

[A propos de l'admission d'un corps en chambre funéraire] "S'il leur manque un papier, ils ne vont pas se refuser le corps. Nous si il manque un papier on ne rentre pas [au sein de la CF]. (...) C'est le problème des papiers, il y a trop de papiers. La famille, au moment d'un décès, il faut qu'elle signe un... deux... déjà deux papiers, simplement pour transporter le corps. Donc à l'hôpital, ils ne vont pas s'amuser... déjà, ils ne savent pas faire. On leur dit de faire signer la demande à la famille, déjà, souvent, ce n'est pas fait...(...) La famille est partie, elle n'a pas signé la demande d'admission, nous on est obligé de lui courir après pour qu'elle signe. Pour eux [gestionnaire de la CF], ce n'est pas grave, elle reviendra bien...(...) Eux ils ont un avantage, c'est qu'ils ne s'imposent pas des règles, ils font ce qu'ils veulent. Ils ne vont pas s'interdire de rentrer un corps, c'est cela qui est important... ".

Par de tels arrangements, le gestionnaire gagne en efficacité opérationnelle, lève la contrainte qui pèse sur ces intervenants et conquiert leur complicité pour de futures opérations (Friedberg, 1993). En revanche, il demeure impitoyable vis-à-vis de ses

\footnotetext{
52 Il s'agit du certificat de décès établi par le médecin, de l'autorisation de transport et de la demande d'admission signés par un membre de la famille.

${ }^{53}$ Le transport d'un corps à l'extérieur de la commune exige, outre les autorisations administratives précédemment citées, la pose d'un bracelet portant l'identité du cadavre par un officier de police.
} 
concurrents, n'hésitant pas à laisser stationner l'ambulance à l'entrée de la chambre funéraire, parfois en pleine nuit, tant que les procédures administratives n'auront pas été accomplies. Sur cette première étape décisive entre le lieu de décès et l'hôtel funéraire, la «fluidité industrielle » est du côté du gestionnaire de la chambre funéraire.

\subsection{Un continuum spatio-temporel et symbolique}

Au sein de l'établissement de santé, première étape du parcours mortuaire, le défunt se trouve aux portes du marché funéraire... Il n'est pas rare qu'une telle représentation ne demeure à jamais théorique pour les principaux intéressés, à savoir ceux qui lui survivent et se chargent de ses funérailles. Dans cette économie de la circulation, l'hétérogénéité des espaces professionnels, les frontières spatiales et les discontinuités temporelles disparaissent au bénéfice d'un perpétuel continuum. Mais là encore, c'est bien l'œuvre de l'opérateur funéraire que de fabriquer des glissières partout où menace la rupture de flux, soit la réouverture du champ des possibles. La pratique de la «double casquette » illustrée par la citation précitée est caractéristique de ce jeu de substitution : derrière l'agent d'état civil, se cache le conseiller funéraire de la régie municipale, qui prolonge immédiatement les formalités administratives par le contrat commercial. Les opérateurs publics (régie, S.E.M.) jouent de multiples façons de leur filiation commune avec ces acteurs publics qui comptent parmi les populations professionnelles les plus fréquemment en contact avec les défunts ou les familles en deuil.

«En mars 1995, une convention a été signée dans «le but de formaliser l'accord aux termes duquel, dès 1945, les Pompes Funèbres municipales avaient tacitement convenu avec l'Assistance publique de $\mathrm{M}^{* * *} \mathrm{~d}^{\text {'installer }}$ un premier poste de régleur à l'hôpital de la Conception, afin que les familles puissent accomplir l'ensemble des formalités administratives, juridiques, consécutives à un décès". La convention précise "qu'eu égard aux résultats obtenus et à la portée d'intérêt général de cette mesure, il fut décidé, à partir des années 1950, de déployer de nouveaux postes de conseillers funéraires sur les sites des hôpitaux Ste $M^{* * *}, M^{* *}$ $\mathrm{L}^{* * *}$, hôpital Nord, $\mathrm{CHU}$ de $\mathrm{T}^{* * *}$ ” (B. O. de la concurrence et de la répression des fraudes, $\mathrm{n}^{\circ} 3$ du samedi 28 février 1998). 
Arrêtons nous une fois de plus à la façon dont se déroule le transit du défunt de l'établissement de santé à la chambre funéraire. La prise en charge immédiate du corps, on l'a évoqué, est souvent providentielle pour l'entourage immédiat du cadavre: médecins, soignants ou même parents. Chambre de l'hôpital, chambre funéraire : d'aucun, encore moins le principal intéressé, ne pose alors la question d'une alternative à ce passage discret d'une chambre à l'autre... Troquant leur sombre costume pour une blouse blanche, les employés des entreprises de pompes funèbres mettent en scène un continuum symbolique entre service hospitalier et service funéraire : "Ma mère a vu arriver deux personnes habillées en blanc avec un brancard. Elle a pensé que c'était du personnel de l'hôpital. Ils ont déposé le corps sur un brancard et l'ont emmené. Elle n'a pas compris qu'il s'agissait déjà des pompes funèbres, non plus que ma grand-mère était transportée à la chambre funéraire. Le personnel soignant lui a ensuite expliqué qu'il ne pouvait pas conserver le corps au sein de la clinique ${ }^{54}$ », raconte une jeune femme interrogée sur le choix d'un opérateur.

En déplaçant le portail d'entrée sur le marché de l'hôpital à la chambre funéraire, le gestionnaire a parcouru l'essentiel du chemin qui le conduit à ses clients. Adossée à la chambre funéraire, l'enseigne de pompes funèbres accueille immédiatement les familles venant reconnaître un corps ou se recueillir auprès du défunt. Maître des lieux, il joue plus aisément sur la contiguïté des espaces, l'interchangeabilité des rôles, la confusion des procédés de signalisation pour réunifier les deux espaces professionnels, le funérarium et l'officine : un hall d'accueil commun, une seule hôtesse à la réception des familles, une ligne téléphonique unique, conduisent inexorablement tout client égaré à la recherche de son proche disparu à rencontrer un agent commercial de l'entreprise. Quand bien même ses concurrents auraient réussi à gagner le mandat d'une famille, il est fort probable que le conseiller commercial intercède auprès de celle-ci pour une offre concurrente et conquiert ses faveurs au bénéfice d'un règlement immédiat du contrat. L'une des règles d'or de l'agence de funérailles, dès lors qu'elle a été mandatée pour transporter un corps à la chambre

\footnotetext{
${ }^{54}$ Dans le cas présent, la clinique dispose d'une petite chambre mortuaire, mais sans personnel affecté directement à sa gestion. Il est donc beaucoup plus confortable pour elle de mobiliser une structure privée.
} 
funéraire, est de ne jamais laisser une famille se rendre sur les lieux avant d'avoir réglé les obsèques.

« [Considérant que] la visite des lieux a mis en évidence l'imbrication des locaux de la chambre funéraire et ceux de l'agence commerciale que $\mathrm{PF}^{\star \star *} \mathrm{y}$ a installée et que la simple mention de «bureau » apposée à la porte, fut-elle fermée, ne permet pas de distinguer ; (...) [Considérant qu'] au terme d'une note de service du 4 mars 1991, dont le sens est exempt d'ambiguïté en raison du but recherché, [que] les entreprises concurrentes se sont vu interdire, pour confirmer la date et l'heure de levée du corps, l'usage du télécopieur; [que] cette interdiction des moyens usuels actuels de communication, en contraignant, de façon discriminatoire, les entreprises concurrentes à dépêcher un employé, sur place, pour procéder à la confirmation requise, a nécessairement eu pour finalité de rendre plus difficile l'exercice de la concurrence... » (B. O. de la concurrence et de la répression des fraudes $n^{\circ} 21$ du vendredi 20 novembre 1998).

Là encore, le juge est régulièrement saisi de la contestation des agences concurrentes qui n'héritent plus que des quelques miettes échappant à ce monopole de fait. Ici ou là, l'heureux gestionnaire de la chambre funéraire est sommé de dresser un mur, sabler une porte vitrée, dissocier les lignes téléphoniques, distinguer le personnel d'accueil. Disjoindre, désunir, séparer : l'action normative tente de rétablir les frontières physiques et l'hétérogénéité des espaces là où l'opérateur funéraire joue sur le «fondu enchaîné ». Elle s'efforce de redonner une visibilité minimale aux agences concurrentes au sein même de la chambre funéraire en imposant l'affichage de la liste des opérateurs... sans préciser cependant qu'il serait préférable que celleci ne soit dissimulée derrière une plante verte...

Restaurer des alternatives, organiser une bifurcation là où le chemin est unique, ouvrir le champ des possibles : tel est l'enjeu de l'opérateur tenu à l'écart de ces itinéraires tracés où circulent les endeuillés, immédiatement précédés par l'être cher disparu. A la conquête active d'une visibilité, s'ajoute la précarité des «attachements ». En laissant transiter le corps par la chambre funéraire, l'opérateur ne risque pas seulement le détournement commercial de son client. Dans la gestion de la relation de service et de la performance productive, il est exposé aux multiples contraintes de coordination que le gestionnaire du funérarium peut lui imposer. 
" * Est-ce qu'ils peuvent faire en sorte que, quand vous utilisez la chambre funéraire, vous ayez des moins bonnes prestations?

- Non... (silence).. Si, si, ils nous filent le salon le plus vilain... et ils nous filent les horaires pour une salle de cérémonie les moins bons, c'est-àdire $8 \mathrm{~h}$ du matin et $17 \mathrm{~h}$ le soir.. Voilà. Ou les horaires de toilette ...(...) Oui, s'ils veulent nous poser des problèmes, c'est leur chambre funéraire, ils sont chez eux : "Tu veux faire l'admission le matin ? Et bien il n'y a personne, tu reviens entre midi et deux ». Et c'est tout ».

L'opérateur est à la discrétion du gestionnaire pour la disponibilité des salons, les horaires d'accueil, l'accès à la salle de soin, la présentation du corps par les employés de la chambre, et toute autre opération se déroulant dans l'enceinte de la chambre funéraire. Ici comme ailleurs, la fluidité industrielle et la qualité de service reposent sur nombre d'arrangements coopératifs dans le quotidien de l'activité, auxquels un concurrent a tout avantage à se soustraire. Si la sociologie du travail et des organisations nous enseigne depuis longtemps à quel point les échanges sont au cœur de l'activité coopérative (Dodier, 1995 ; Friedberg, 1993), la sociologie du marché rencontre immédiatement la prégnance de l'activité coopérative au cœur de la dynamique concurrentielle.

\subsection{Hyper-coordination et engagement}

Nous sommes loin d'avoir appréhendé l'infinie variété de ces dispositifs d'aiguillage dont les professionnels des services funéraires ont le secret. Mais nous sommes maintenant suffisamment familiarisés avec cette grammaire de la coordination marchande pour tenter maintenant de mieux la qualifier.

Le cadrage de la circulation est au cœur de cette économie de la captation. En suivant les défunts et leurs proches, on découvre les multiples ressorts du travail de formatage des itinéraires de circulation : les techniques d'adressage (commutation automatique, signalisation), l'encadrement spatio-temporel des flux (proximité spatiale, rapidité d'intervention), l'attachement de produits ou prestations hétérogènes (état civil/vente ; soins au mourant / soins au défunt), la continuité symbolique (blouse blanche), les échanges et arrangements coopératifs avec les multiples protagonistes de l'activité et plus généralement la connexion étroite 
d'espaces professionnels distincts (chambre hospitalière - chambre funéraire enseigne commerciale). La fluidité des enchaînements est essentielle: elle se fabrique par «l'hyper coordination» (Thrift, 2003), soit par la configuration d'agencements sociotechniques qui « conditionnent les positions et juxtapositions ».

Si l'architecture matérielle de la canalisation est essentielle, il faut également réussir «l'enrôlement » des multiples intermédiaires au sein des réseaux de circulation. En amont, policier, soignant, agent d'amphithéâtre, précèdent l'opérateur dans la rencontre avec les défunts. En matière de structuration du marché, la sociologie économique a coutume de désigner ces intermédiaires de «prescripteurs » (Hatchuel, 1995), soit des figures actives du processus de qualification des produits. Mais il faut ici leur reconnaître une qualité plus essentielle : celle de se situer directement, à un titre ou un autre, sur ces itinéraires de circulation des corps et/ou des familles, et davantage d'être en charge d'en réguler la gestion ou le flux. Car c'est bien relativement aux impératifs de coordination et de gestion des flux que s'engagent des arrangements coopératifs privilégiés avec certains opérateurs funéraires.

«L'économie des biens cède la place à une économie des liens » (Callon, et al., 2000 p.238) La stabilisation des réseaux sociotechniques mobilisés dans le process concentre le jeu concurrentiel. On aura constaté à quel point la forte polarité de la chambre funéraire, équipement central de la relation de service, rend extrêmement précaire l'existence de liens alternatifs à celui qui va irrémédiablement s'établir avec les pompes funèbres qui résident sur les lieux, y compris ex post, via les entraves à la qualité de prestation de leurs concurrents.

La portée captatrice de ces techniques de rencontre est aussi liée à leur capacité à fabriquer des «engagements », dans une acception proche de celle développée par H. Becker (Becker, 1960) ${ }^{55}$. Le défunt réduit au silence, la famille en proie à

\footnotetext{
${ }^{55}$ L'auteur rapporte la notion «d'engagement » à l'effet à terme de décisions ou d'action initiales prises dans un contexte donné, qui imposent au-delà de ce contexte une ligne de conduite, un enchaînement des situations, une «carrière » : «It seems convenient to retain «commitment » to refer to the specific mechanism of constraint of behavior through previously placed side bets and use terms as «involvement », «attachment», «vocation», « obligation», and so on, to refer to related but
} 
l'urgence, le soignant pressé de se débarrasser des corps : tous se laissent volontiers absorbés par la mise en œuvre d'une capacité sociotechnique par le professionnel funéraire, étroitement ajustée au système de contrainte que déclenche le décès. L'engagement est d'abord pratique: il traduit cette propriété des agencements sociotechniques à figer la trajectoire des défunts. Une fois le défunt enrôlé malgré lui dans cet itinéraire cadré, il ne suffit pas de réouvrir le champ des possibles, ne seraitce qu'à la conscience des familles, pour relancer le jeu du marché. Parce qu'elle précède l'établissement du contrat commercial, la relation de service - entendue comme allocation de moyens associés au traitement du défunt - produit de l'engagement. Toute démarche alternative engage pour le client un coût supplémentaire important : cela suppose qu'il décline l'offre du prestataire après que celui-ci se soit mobilisé activement à son service, qu'il règle ses dettes pour repartir en quête d'un autre prestataire, qu'il explore des alternatives, qu'il se déplace souvent en nombre et donc de concert - d'une boutique à l'autre, qu'il ait la disponibilité de réengager un entretien d'au moins quarante-cinq minutes avec un autre conseiller, qu'il se prive d'une offre qui pouvait finalement bénéficier de sa préférence, etc.

De l'établissement de soin à la chambre funéraire, de la chambre funéraire au local commercial des pompes funèbres : les ressorts de la mise en relation du client et du prestataire empruntent leur dynamique à ces modèles séquentiels de la carrière qu'affectionnent les interactionnistes ou encore aux processus d'auto-renforcement chers aux théoriciens des «sentiers de dépendance ». «In path-dependent sequences, the key mechanism at work is some form of self-reinforcement or positive feedback loop. Initial moves in a particular direction encourage further movement along the same path. Over time, "the road not chosen" becomes increasingly distant, increasingly unreachable alternative" (Pierson, 2000b, p. 74-75) ${ }^{56}$. Actes ou choix

distinguishable phenomena ». Sur la notion d'engagement comme ligne d'action conséquente à une action initiale, voir également (Johnson, 1973).

56 A partir d'une revue de la littérature en économie et sciences politiques, l'auteur redéfinit les fondements analytiques du concept de «Path Dependence »: qu'il rapporte aux processus sociaux fondés sur une dynamique de rendements croissants. Il s'efforce ensuite de mobiliser cette clé d'analyse dans la compréhension de phénomènes centraux de la vie politique (action collective, dynamiques institutionnelles, autorité politique et asymétrie de pouvoir, etc.). 
initiaux déterminent une ligne d'action, à ceci près que le poids de la détermination est ici rigoureusement orchestré par les acteurs du marché.

\section{Conclusion}

Qu'ils se postent à l'entrée des mairies, des cimetières ou à la sortie des hôpitaux, les pompes funèbres partent à la rencontre des clients qui ne viennent pas d'eux-mêmes visiter leurs boutiques. ... Guetter à la croisée des chemins, formater les itinéraires de circulation du client, s'approprier les corps pour attraper les familles, organiser la «fluidité industrielle » par le maillage étroit d'entités hétérogènes, autant de façons d'œuvrer pour substituer à la complexité du jugement en situation de concurrence un chemin tout tracé vers un opérateur unique.

Comprendre l'organisation du marché, c'est donc, entre autres, s'intéresser à la circulation des clients, aux dispositifs sociotechniques qui anticipent et structurent leurs itinéraires, aux agencements symbolico-matériels qui fabriquent les chemins tracés de la rencontre marchande. L'étude des stratégies et conflits de concurrence entre opérateurs funéraires sur les marchés locaux nous met directement en prise avec cette autre modalité d'organisation de la relation offre/demande que celle classiquement rapportée à l'activité de calcul et de qualification : les équipements de la relation marchande, tout au moins ceux qui structurent le processus de mise en relation d'un client et d'un prestataire, relèvent moins de l'économie du jugement que de celle de la captation, en organisant la circulation du client. Dans cette économie, le gouvernement des réseaux sociotechniques associés au process, notamment à l'amont de la filière, constitue un levier puissant de construction d'une position dominante sur le marché.

Au-delà de la spécificité du contexte funéraire, on perçoit la portée plus générale de tels équipements du marché. Ils ont un poids d'autant plus important dans l'organisation de la relation marchande que le client est pris dans une économie du temps, voire de l'urgence, ou encore disposé à échapper au travail cognitif d'identification et de classification d'offres concurrentes. Ces dispositifs constituent des composantes à part entière de la dynamique des marchés. Leur performance tient 
avant tout à leur capacité à organiser la raréfaction des rencontres entre clients et prestataires (Callon, Muniesa, 2003), à substituer à l'espace de choix du marché une orientation déterminée du client.

Cette lecture suggère l'intérêt de nous doter de catégories conceptuelles qui permettent de spécifier analytiquement les différents mécanismes à l'œuvre dans la construction de la rencontre marchande, notamment autour de la distinction entre instrumentation de la qualification et instrumentation de l'engagement. D'un côté, le mécanisme clé du processus de rencontre s'élabore autour de la définition des propriétés et des qualités de l'objet de l'échange. De l'autre, il s'accomplit par la construction et le renforcement de l'irréversibilité des choix initiaux (Pierson, 2000a). Cette proposition suppose de prêter une plus grande attention à ces versions microsociologiques des «sentiers de dépendance » qui participent à la dynamique d'organisation des marchés. Car elles donnent accès à l'infinie diversité des dispositifs à partir desquels les acteurs du marché structurent des processus d'enchaînement, produisent de l'inertie excluant le jeu des alternatives, comptent et composent avec les lois qui gouvernent les chemins de dépendance : le rôle critique et performatif des séquences initiales, la centralité des facteurs spatiaux et temporels, le poids de la coordination au sein des réseaux sociotechniques attenant au processus de production. 


\section{BIBLIOGRAPHIE CITEE}

Auby J. F., 1997. - Les obsèques en France, Paris, P. U. F.

Barrau A., 1987. - «Les services funéraires, Permanences et évolutions », Economie et statistique, 198.

Baudry B., 1994. - « De la confiance dans la relations de sous-traitance », Sociologie du travail, XXXVI, 1, 43-61.

Baudry P., 1995. - « Devant le cadavre », Religiologiques, 12, 19-29.

Becker H., 1960. - « Notes on the Concept of Commitment», American journal of sociology, 66, 32-40.

Boissin O., Trompette P., 2004. - «La libéralisation des services funéraires: des régulations qui se cherchent », Economies et sociétés, 24, 11, 1933-1960.

Boltanski L., Thévenot L., 1987. - Les économies de la grandeur, Paris, P. U. F.

Callon M., 1986. - «Eléments pour une sociologie de la traduction: la domestication des coquilles St Jacques et des marins-pêcheurs dans la baie de St Brieux », L'année sociologique, 36, 169-208.

Callon M. (dir), 1998 The laws of the markets, Oxford, Blackwell Publishers.

Callon M., Meadel C., Rabeharisoa V., 2000. - «L'économie des qualités », Politix, 13, 52, 211-239.

Callon M., Muniesa F., 2003. - «Les marchés économiques comme dispositifs collectifs de calcul », Réseaux, 21, 122, 189-233.

Cochoy F., 2002. - L'âne de Buridan et l'économie du package. Essai d'anthropologie du marché. Paris, PUF.

Cochoy F. d. (dir), 2004 La captation des publics. C'est pour mieux te séduire, mon client... Toulouse, Presses Universitaires du Mirail.

Cusin F., 2004. - « Attirer, sélectionner, fidéliser: le double marché du crédit aux particuliers », in F. Cochoy (dir), La captation des publics. C'est pour mieux te séduire, mon client... Toulouse, Presses Universitaires du Mirail.

d'Abbadie G., 2003. - « Dix ans après la loi Sueur, quels régulateurs pour le secteur funéraire », L'Officiel du Funéraire, numéro spécial février 2003, 5-8.

De Bandt J., Gadrey J., 1994. - Relations de service, marchés de service, Paris, CNRS Editions.

Dodier N., 1995. - Les hommes et les machines, Paris, Métailié.

Dubuisson-Quellier S., 1999. - « Le prestataire, le client et le consommateur.

Sociologie d'une relation marchande », Revue française de sociologie, XL, 4, 671688.

Dunham A., 1963. - « The method, process and frequency of wealth transmission at death », University of Chigago law review, 30, 241-285.

Eymard-Duvernay F., Marchal E., 1997. - Façons de recruter. Le jugement des compétences sur le marché du travail, Paris, Métailié.

Favereau O., 1989. - «Organisation et marché », Revue française d'économie, 4, 1, 65-96. 
Friedberg E., 1993. - Le pouvoir et la règle. Dynamique de l'action organisée, Paris, Seuil.

Garcia M.-F., 1986. - «La construction sociale d'un marché parfait : le marché au cadran de Fontaines-en-Sologne », Actes de la recherche en sciences sociales, 65, 213.

Hatchuel A., 1995. - «Les marchés à prescripteurs: crises de l'échange et genèse sociale », in A. Jacob et H. Verin (dirs), L'inscription sociale du marché, Paris, L'Harmattan.

Henaff M., 2002. - « L'argent et le hors-de-prix », Esprit, 2, 166-177.

Johnson M., 1973. - « Commitment: a conceptual analysis and empirical application », Sociological Quaterly, 14, 395-406.

Karpik L., 1989. - «L'économie de la qualité », Revue française de sociologie, $X X X$, 187-210.

Latour B., 1989. - La science en action, Paris, La Découverte.

Licoppe C., Pharabod A.-S., Assadi H., 2002. - «Contribution à une sociologie des échanges marchands sur internet », Réseaux, 20, 116, 99-140.

Musselin C., Paradeise C., 2002. - « La qualité », Sociologie du travail, 44, 255287.

Pierson P., 2000a. - «Increasing Returnes, Path Dependence, and the Study of Politics », American Political Science Review, 94, 2, 251-267.

Pierson P., 2000b. - « Not Just What, but When : Timing and Sequence in Political Processes », Studies in American Political Development, 14, 72-92.

Riveline C., 1991. - «De l'urgence en gestion», Gérer et comprendre, 22, 82-92.

Rot G., 2002. - «Fluidité industrielle, fragilité organisationnelle », Revue Française de Sociologie, 43, 4, 711-737.

Rubat du Merac H., 1905. - Le nouveau régime des pompes funèbres, Paris, Lethielleux.

Salais R., 1989. - «L'analyse économique des conventions de travail », Revue économique, 40, 2, 199-240.

Salais R., Thévenot L., 1986. - Le travail, marchés, règles, conventions, Paris, Economica.

Segrestin D., 1997. - «L'entreprise à l'épreuve des normes de marché. Les paradoxes des nouveaux standards de gestion dans l'industrie », Revue Française de Sociologie, $38,3,553-585$.

Théodore-Vibert P., 1905. - Le privilège catholique des pompes funèbres et la séparation des Eglises et de l'Etat, Foix, Imprimerie Gadrat Ainé.

Thomas L. V., 1980. - Le cadavre, De la biologie à l'anthropologie, Bruxelles, Editions Complexe.

Thompson E. W., 1991. - « Handling the stigma of handling the dead: morticians and funeral directors », Deviant behaviour: an interdisciplinary journal, 12, 403-429. Thrift N., 2003. - Remembering the technological unconsious by foregrounding kmowledges of position, Bristol: University of Bristol.

Trompette P., Boissin O., 2000. - «Entre les vivants et les morts: les pompes funèbres aux portes du marché. », Sociologie du travail, 42, 3, 483-504.

Vatin F., 1987. - La fluidité industrielle, Paris, Méridiens Klincksieck. 
Vinck D., 1999. - «Les objets intermédiaires dans les réseaux de coopération scientifique. Contribution à la prise en compte des objets dans les dynamiques sociales », Revue Française de Sociologie, XL, 2, 385-414.

Zaichkowsky J. L., 1985. - « Measuring the involvement construct», The journal of consumer research, 12, 3, 341-352.

Zelizer V. A., 1978. - « Human values and the market: The case of life insurance and death in 19th-century America », American journal of sociology, 84, 3, 591-606. 